\title{
Actitudes del profesorado de Educación Física hacia la inclusión de alumnos con discapacidad Physical Education teachers' attitudes towards the inclusion of students with disabilities
}

\author{
*Patricia Solís García, **Víctor Borja González
}

*Universidad Internacional de la Rioja (España), **Colegio Sta. María del Naranco Alter-Via (España)

\begin{abstract}
Resumen. El modelo educativo basado en la inclusión refleja las necesidades sociales y se basa en la educación de calidad para todos los alumnos y la atención de sus necesidades particulares. Los docentes juegan un papel crucial en el desarrollo de metodologías inclusivas y su actitud va a determinar en gran medida el éxito de dichas metodologías y del propio modelo inclusivo. Con el objetivo de conocer las actitudes hacia la inclusión educativa de alumnado con discapacidad en maestros de educación física de primaria y secundaria, se examinan datos provenientes de 72 docentes de esta área (60\% hombres y $40 \%$ mujeres), que ejercen tanto en centros escolares públicos como privados en varias comunidades autónomas del estado español. Se ha utilizado un cuestionario para profesores de actitudes hacia los alumnos con necesidades educativas especiales derivadas de discapacidad. Los resultados muestran que los profesores presentan actitudes moderadamente positivas hacia la inclusión de alumnos con discapacidad. Se concluye la necesidad de optimizar la formación en materia de inclusión y la realización de programas de prácticas que incluyan contacto con el alumnado con discapacidad.

Palabras clave: profesores, educación física, actitudes, inclusión, discapacidad.
\end{abstract}

Abstract. The educational model based on inclusion reflects social needs and is based on quality education for all students and attention to their particular needs. Teachers play a crucial role in the development of inclusive methodologies and their attitude largely determines the success of these methodologies and the inclusive model itself. In order to assess physical education teachers' attitudes toward educational inclusion of students with disabilities in primary and secondary education, data from 72 teachers in this area $(60 \%$ men and $40 \%$ women), who work both in public and private schools in several autonomous communities of Spain, are examined. A questionnaire on teachers' attitudes towards students with special educational needs derived from disability was used. The results show that teachers have moderately positive attitudes towards the inclusion of students with disabilities. The need to optimize training in inclusion and the realization of practical programs that include contact with students with disabilities is concluded.

Keywords: teachers, physical education, attitudes, inclusion, disability.

\section{Introducción}

Los modelos educativos vigentes buscan dar una respuesta a los desafíos de la sociedad actual, y en este sentido la normalización de personas con discapacidad es una prioridad en las sociedades avanzadas (Novo-Corti, Muñoz-Cantero \& Calvo-Babío, 2015). En las aulas del siglo XXI se debe aceptar el reto de enseñar a una población estudiantil diversa, incluyendo aquellos con discapacidad (Hodge, Lieberman \& Murata, 2017), ya que la inclusión de estos alumnos es claramente el futuro de educar a este colectivo (Block, Kwon \& Healy, 2016) así como un derecho firmado por las Naciones Unidas (UNESCO, 2005). Este reto se refiere específicamente a la escuela inclusiva vinculada a una educación de calidad sin excepciones, y una filosofía que contempla la educación como motor de cambio social y de actitudes (Ríos, 2009).

Han pasado ya 15 años desde que la UNESCO definiera la inclusión como «Un proceso de abordaje y respuesta a la diversidad de las necesidades de todos los alumnos a través de la creciente participación en el aprendizaje, las culturas y las comunidades, y de la reducción de la exclusión dentro y desde la educación. Implica cambios y modificaciones en los enfoques, las estructuras, las estrategias, con una visión que incluye a todos los niños de la franja etaria adecuada y la convicción de que es responsabilidad del sistema regular educar a todos los niños» (UNESCO, 2005, p. 13).

De esta definición se desprende la importancia de ser culturalmente receptivo y adquirir el conocimiento necesario

Fecha recepción: 09-02-20. Fecha de aceptación: 01-05-20

Patricia Solís García

patricia.solis@unir.net para infundir prácticas apropiadas y socialmente justas en entornos educativos (Hodge et al., 2017). Así la escuela inclusiva se fundamenta en la premisa de que las dificultades de aprendizaje se relacionan estrechamente con las diversas manifestaciones de la organización escolar (Ríos, 2009) y, por tanto, son las propias carencias del sistema educativo a la hora de conseguir la necesaria y total inclusión de los alumnos con discapacidad en las aulas las causantes de la falta de dicha inclusión (González \& Cortés, 2016). Por ello, el vínculo entre discapacidad y marginalización en la educación sigue siendo evidente en todos los países, independientemente de su grado de desarrollo (Novo-Corti et al., 2015).

Si buscamos aplicar el modelo inclusivo al área de Educación Física (EF), «todo el alumnado comparte el mismo espacio, sin diferencias, reconsiderando la enseñanza y su organización con el apoyo pedagógico y social que sea necesario, y manteniendo las más altas expectativas para el aprendizaje de todas y todos.» (Ríos, 2009, p.88). Hay evidencias de la relevancia que tiene la práctica de EF para la inclusión de los alumnos con discapacidad y sus iguales (González \& Cortés, 2016) siendo esta área un contexto idóneo para la creación de entornos de aprendizaje inclusivos (Felipe-Rello, Garoz \& Tejero-Gonzalez, 2018; Muñoz, Smith \& Matos, 2020) facilitando la transmisión de valores y actitudes relacionados con la normalización, la igualdad y la inclusión social (Felipe-Rello, Garóz, \& Tejero-González, 2020). Asimismo, la actividad física desde el área de EF predice un mayor autoconcepto en escolares de 8 a 12 años (Guillamón, García \& Carrillo, 2019), en adolescentes (Gentil,Zurita, Gómez, Padial \& Lara, 2019) y específicamente en alumnos con discapacidad motriz (Fernández, Jiménez, Navarro \& Sánchez, 2019). No obstante, muchas investigaciones confirman que 
el alumnado con discapacidad realiza una actividad física significativamente menor que sus compañeros en la clase de EF (Abdi, 2017). Rekaa, Hanisch \& Ytterhus (2019) realizan una revisión al respecto y hallan que los estudiantes con discapacidad experimentan exclusión y falta de pertenencia en EF. Se sugiere así que estos estudiantes corren el riesgo de ser rechazados debido a las expectativas inalcanzables de los docentes (Reich, 2012) y es que los docentes se han enfrentado al reto de la atención de estudiantes con discapacidad en aulas inclusivas y a la necesidad de brindarles apoyo y EF adaptada (Hutzler, Meier, Reuker \& Zitomer, 2019). Muchos de ellos sienten que incluir a los estudiantes con discapacidad les proporciona beneficios, pero señalan asimismo que les preocupan las dificultades prácticas de enseñar a estos estudiantes, la falta de apoyo y el posible rechazo a los estudiantes con discapacidad por parte de sus compañeros (Wang, Qi \& Wang, 2015).

En este sentido, los docentes juegan un papel decisivo para hacer realidad la educación inclusiva (Jerlinder, Danermark \& Gill, 2010). Así las cosas, se observa que, en los últimos años, una parte vital para implementar una inclusión exitosa son las actitudes y la autoeficacia del docente (Reina, Ferriz \& Roldán, 2019, Reina, Santana, Montesdeoca \& Roldán, 2019). Comprender las perspectivas de los docentes sobre la inclusión es crucial en la investigación educativa (Abdi, 2017), más aún quizás el factor más importante para una inclusión exitosa en EF es la actitud y la competencia percibida de los docentes de EF (Block et al., 2016) porque las actitudes son un determinante importante del comportamiento de los docentes (Cyran, Kudlacek, Block, MalinowskaLipien \& Zyznawska, 2017).

La primera definición de actitud la encontramos en Allport (1935), esta se refiere a un estado que predispone al organismo ante una acción determinada que se aprende y organiza mediante la experiencia. Triandis (1971, p.2) entiende la actitud como «una idea provista de una carga emocional que predispone a una clase de acciones ante un determinado tipo de situaciones sociales». En la investigación que se presenta se opta por el modelo de los tres componentes de las actitudes, a saber: componente cognitivo (ideas, creencias, opiniones o percepciones acerca del objeto), componente afectivo (expresado a través de los sentimientos agradables o desagradables) y componente conativo-conductual (predisposición para actuar). La actitud aglutina por tanto las valoraciones realizadas por una persona sobre los objetos o estímulos de su entorno y conlleva, modos de pensar, sentir y actuar más o menos estables ante tales estímulos. En el ámbito educativo que plasmamos, es de presuponer que a mayor actitud positiva del profesorado mayor uso de estrategias que faciliten la inclusión (Garzón, Calvo \& Orgaz, 2016, Pegalajar \& Colmenero, 2014).

A este respecto, revisando estudios previos sobre actitudes de los docentes de EF hacia la inclusión de alumnos con discapacidad encontramos que en su mayoría estos muestran actitudes positivas (Abellán, 2015) aunque debemos ser conscientes de que las actitudes hacia la inclusión son un constructo complejo que varía entre países y entre escuelas (Di Nardo, et al., 2014). Dichas actitudes se basan en ocasiones en el nivel de participación que los niños con discapacidad pueden lograr (Morley, Bailey, Tan \& Cooke,
2005) y reflejan asimismo el desafío que supone el diseño de sesiones inclusivas para estos alumnos especialmente en el caso de discapacidades severas (Hodge et al., 2017; Sato y Hodge, 2009). Se encuentra que a menudo los docentes de EF no se sienten preparados o lo suficientemente seguros de sí mismos para implementar con éxito esta inclusión(Block et al., 2016; Hutzler et al., 2019). Muchos perciben la inclusión imposible de lograr debido a la falta de competencia y la falta de recursos y herramientas (Valencia-Peris, MínguezAlfaro \& Martos-García, 2020) y la experiencia docente también condiciona la actitud del profesorado (González \& Macías, 2018). Pero una barrera actitudinal principal es la presuposición del estudiante de EF «normativo» construido (Rekaa et al., 2019). Una da las razones de esto podría ser la falta de formación inicial o permanente sobre el tema (Fernández-Rivas \& Mateos, 2017). Los docentes expresan la necesidad de más capacitación profesional orientada específicamente a la enseñanza de estudiantes con discapacidades severas, trastornos de conducta emocional, hiperactividad y déficit de atención (Casebolt \& Hodge, 2010). En síntesis, Huztler et al., (2019) señalan que partiendo de una perspectiva práctica, los profesionales de la inclusión educativa deben ser conscientes de varios factores que influyen en las actitudes al participar en la inclusión del alumnado con discapacidades en EF: (a) Si ha tenido o no experiencia previa y la calidad de ésta; (b) La formación profesional y académica respecto a la inclusión; (c) Factores individuales, incluido el sexo; (d) Factores ambientales de la escuela, como un proceso en lugar de una orientación al desempeño; y, finalmente, (e) El tipo y grado de discapacidad.

Un factor primordial facilitador de la inclusión educativa es el conocimiento de las opiniones y actitudes del profesorado ante las respuestas educativas que despliegan los centros escolares (Domínguez-Alonso, López-Castedo, Pino \& Vázquez, 2015; Torres \& Fernández, 2015). Sin embargo, existe una investigación limitada sobre cómo los docentes perciben y ayudan a los estudiantes a participar en la EF (Abdi, 2017), por ello el presente estudio tiene como objetivo conocer las actitudes del profesorado de EF hacia la inclusión del estudiantado con discapacidad en lo referente a la responsabilidad del profesor, el rendimiento de los alumnos, la formación y recursos disponibles, el clima del aula y el desarrollo social de los alumnos, contrastando dichas actitudes según el tipo de centro educativo y ubicación de éste, sexo, edad y años de experiencia como docentes.

\section{Método}

Para lograr describir sistemáticamente las características presentes en la población estudiada se ha utilizado una metodología descriptiva. Se exponen seguidamente el instrumento, participantes y procedimiento utilizados para la obtención de resultados.

\section{Instrumento}

El instrumento de medida utilizado para conocer las actitudes del profesorado hacia los alumnos con necesidades educativas especiales derivadas de discapacidad es de Mula (2002), en la versión adaptada y validada por Solís, Pedrosa y Fernández-Mateos (2019). Dicho instrumento aborda 5 di- 
mensiones con índices de ajuste aceptables relacionadas con las actitudes en 22 ítems con un formato de respuesta tipo Likert en donde 1 indica «Muy en desacuerdo» y 5 «Muy de acuerdo». Dichas dimensiones son Responsabilidad (4 ítems) (p. ej. Creo que la formación de los alumnos con discapacidad es responsabilidad de los especialistas) Rendimiento (3 ítems) (p. ej. El reto que representa estar en una clase ordinaria estimula el desarrollo académico del alumno con discapacidad), Formación y Recursos (6 ítems) (p. ej. Creo que el centro ofrece la suficiente formación para que los profesores amplíen su conocimiento sobre la discapacidad), Clima del aula (3 ítems) (p. ej. Es difícil mantener el orden y disciplina en una clase ordinaria a la que asisten alumnos con discapacidad) y Desarrollo social (5 ítems)( p. ej. La imagen social de los alumnos con discapacidad tal y como la ven los compañeros, mejora a través de la interacción con el grupo).

Se recogen también datos concernientes a las cuestiones sociodemográficas relativas al profesorado y al centro educativo en el cual desempeña su labor docente.

\section{Participantes}

Los docentes participantes en este estudio componen una muestra de 72 profesores de EF (60\% hombres y 40\% mujeres), los cuales desempeñan su función en colegios públicos $(76 \%)$ y concertados de diversas provincias de España. La gran mayoría (89\%) imparte docencia en educación primaria y el $11 \%$ lo hace en secundaria, y en centros urbanos (58\%) y rurales. En lo que respecta a su edad, esta oscila en un rango de los 25 a los 53 años $(M=36.38$, DT=6.44) con una trayectoria docente variada $(\mathrm{M}=8.33, \mathrm{DT}=5.66)$ y el $65 \%$ declaran tener un contacto previo con alumnos con discapacidad.

\section{Procedimiento}

En primer lugar, se procedió a un contacto inicial con los equipos directivos de diversos centros escolares para informar sobre la naturaleza y objetivos de investigación. Una vez mostraron su disposición a colaborar se facilitó un enlace digital al cuestionario a fin de que el profesorado que mostrase interés en la investigación pudiese cumplimentar anónimamente dicho cuestionario, sin que mediase recompensa alguna y cumpliendo con los requisitos exigidos por el Comité de Ética en Investigación tratando de evitar los potenciales sesgos en las respuestas.

Tras la recopilación de datos estos fueron analizados con el software SPSS que permite la realización de pruebas estadísticas no paramétricas que determinen la existencia de diferencias estadísticas significativas en las cinco dimensiones evaluadas y las variables respecto a las características sociodemográficas del profesorado (sexo, edad y experiencia docente) y centro educativo (público/concertado, urbano/rural). Dado el reducido tamaño de la muestra se han realizado pruebas paramétricas con muestra independientes aplicando la U de Mann-Whitney ( $\mathrm{NC}=95 \%)$.

\section{Resultados}

La muestra está formada por docentes que difieren en cuanto a determinadas características, por este motivo se pretende averiguar la existencia de diferencias estadísticamente significativas que afectan a las actitudes del profesorado de EF hacia la inclusión de alumnos con discapacidad en sus clases (relacionadas con el tipo y ubicación del centro, sexo, edad y experiencia docente).

Explorando a grandes rasgos las actitudes generales de los docentes participantes (figura 1) estas son moderadamente positivas situándose en todas las dimensiones por encima de los 3 puntos. La actitud más positiva se da en la dimensión relación social en la cual se alcanza una media de 4.3, en el extremo contrario se sitúa la dimensión responsabilidad en la cual se obtiene una media de 3.3 puntos. Debido a la naturaleza descriptiva del trabajo y a que el grupo de participantes se puede considerar uno solo se ha realizado un Test de Friedman para comparar las diferencias en las cinco dimensiones. Se concluye que existen diferencias significativas en las diferentes dimensiones $\left(\mathrm{X}^{2}(4)=230.42, p<\right.$ $0.05)$.

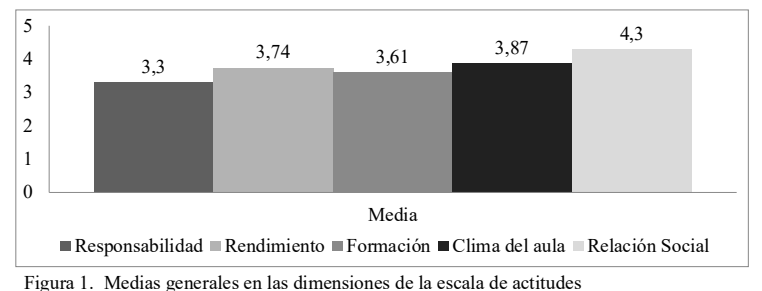

Pasando al análisis de las diferencias en función de las variables predictoras y comenzando con las relativas al tipo de centro (tabla 1), no se hallan diferencias en función del tipo de centro (público/privado) si bien los rangos promedios son más altos en todas las dimensiones para los docentes que ejercen su profesión en centros públicos. En cuanto al ámbito donde se ubica el centro, se encuentran diferencias en las dimensiones rendimiento, formación y recursos y relación social, mostrando los rangos promedios más elevados los docentes que trabajan en centros de ámbito rural.

\begin{tabular}{|c|c|c|c|c|c|c|}
\hline & \multicolumn{3}{|c|}{ Tipo de centro } & \multicolumn{3}{|c|}{ Ámbito de centro } \\
\hline & $U$ & $Z$ & $p$ & $U$ & $Z$ & $p$ \\
\hline Responsabilidad & 331.50 & -1.81 & .06 & 480.00 & -1.72 & .08 \\
\hline Rendimiento & 359.00 & -1.44 & .14 & 411.00 & -2.51 & $.01 *$ \\
\hline Formación y recursos & 364.50 & -1.36 & .17 & 376.50 & -2.90 & $.00^{*}$ \\
\hline Clima del aula & 410.00 & -.77 & .44 & 470.50 & -1.84 & .06 \\
\hline Relación social & 351.00 & -1.57 & .11 & 399.00 & -2.69 & $.00^{*}$ \\
\hline
\end{tabular}

En lo relativo a las variables que caracterizan al profesorado, no se encuentran diferencias estadísticamente significativas en función del sexo, experiencia docente o edad. No obstante, centrándonos en el sexo, las mujeres presentan rangos más altos en todas las dimensiones a excepción de la dimensión formación y recursos en la que los hombres presentan rangos más altos. En cuanto a la experiencia docente (menos de 10 años o 10 o más años de trayectoria), encontramos que los docentes más noveles presentan rangos más altos en todas las dimensiones, sin embargo, en lo referente a los años (menos de 35 o 35 o más años) encontramos rangos más altos en todas las dimensiones salvo en la relativa a clima del aula con una mínima diferencia a favor de los docentes más jóvenes. 
Tabla 2.

\begin{tabular}{|c|c|c|c|c|c|c|c|}
\hline & \multicolumn{2}{|c|}{ Sexo } & \multicolumn{3}{|c|}{ Experiencia docente } & \multicolumn{2}{|c|}{ Edad } \\
\hline & $U$ & $p$ & $U$ & $Z$ & $p$ & $U$ & $Z$ \\
\hline Responsabilidad & $584.50-.45$ & .65 & 633.00 & -.29 & .97 & 531.50 & -1.19 \\
\hline Rendimiento & $563.50-.69$ & .48 & 549.00 & -.99 & .32 & 617.00 & -.21 \\
\hline Formación y recursos & $540.50-.95$ & .34 & 583.50 & -.59 & .55 & 564.50 & -.80 \\
\hline Clima del aula & $600.50-.26$ & .78 & 593.00 & -.49 & .62 & 625.50 & -.11 \\
\hline Relación social & $601.00-.26$ & .79 & 537.00 & -1.14 & .25 & 618.50 & -.19 \\
\hline
\end{tabular}

\section{Discusión}

El modelo educativo del siglo XXI basado en la atención a la diversidad de todo el alumnado persigue la inclusión educativa como criterio de calidad. La inclusión exitosa se basa en la cooperación y atención a las necesidades del alumnado en todas las áreas del currículo, así las actitudes y la autoeficacia de los futuros maestros de EF se consideran uno de los parámetros más importantes para una inclusión exitosa (Jovanoviæ, Kudláèek, Block \& Djordjeviæ,2014). El caso particular de inclusión en EF plantea un desafío específico para la práctica docente (Jerlinder et al., 2010). Dicho desafío se ve en gran medida influido por las actitudes de los docentes que guían su conducta. En este sentido, la investigación sobre las actitudes de los maestros de EF hacia sus estudiantes con discapacidades es extremadamente limitada (Abdi, 2017; Reich, 2012). Por este motivo, el objetivo perseguido con este estudio es conocer las actitudes del profesorado de EF hacia la inclusión de alumnos con discapacidad, contrastando dichas actitudes según el tipo de centro educativo y ubicación de este, sexo, edad y años de experiencia como docentes.

A la luz de los resultados estudiados, las actitudes generales hacia la discapacidad del profesorado de EF que ha formado nuestra muestra son moderadamente positivas. Este hecho coincide con otros estudios realizados con profesorado en general (Cagran \& Schmidt, 2011; Dias \& Cadime, 2016; Rakap, Parlak-Rakap \& Aydin, 2016; Saloviita \& Schaffus, 2016; Urton, Wilbert \& Hennemann, 2014) y con especialistas en EF (Jerlinder et al., 2010; Reich, 2012; Wang et al., 2015). En nuestro estudio los docentes han mostrado una actitud altamente positiva en la dimensión de relación social, hecho a destacar dadas las posibilidades de cooperación y formación de la identidad personal que se originan en las clases de EF. La educación en actitudes y valores será la base para que todo el alumnado respete la diversidad (Ríos, 2009).

Debemos considerar la idiosincrasia y particularidades de los docentes, Ríos (2009, p.91) lo señala como factor a tener en cuenta, «las experiencias previas, su propia ideología, su actitud hacia la búsqueda de la solución de problemas, su capacidad de innovación, entre otras, pueden determinar las posibilidades educativas del alumnado y, por tanto, situarlo en mejores o peores condiciones para el aprendizaje.» Por ello, se ha procedido al análisis de las diferencias en función de las variables sociodemográficas de la muestra. En nuestro caso no se encuentran diferencias estadísticamente significativas en función del sexo, experiencia docente o edad lo cual coincide con los estudios de Jerlinder et al. (2010) y Wang et al. (2015) salvo que en ambos sí hallan una diferencia: los docentes de EF con experiencia previa real en la enseñanza de alumnos con discapacidad se muestran ligeramente más positivos hacia la EF inclusiva. Jovanoviæ et al. (2014) encuentran además diferencias respecto al sexo, mostrando las mujeres actitudes más positivas.

En lo que respecta al tipo de centro, en nuestro estudio no se encuentran diferencias estadísticamente significativas en cuanto a la titularidad del centro, pero sí en cuanto al ámbito donde se ubica el centro. Mostrando actitudes más positivas en las dimensiones rendimiento, formación y recursos y relación social los docentes que trabajan en centros de ámbito rural. No hemos podido contrastar este hallazgo ya que no hemos rescatado estudios previos que analicen el ámbito en el cual se enclava el centro escolar.

La implementación práctica y exitosa de la inclusión aún requiere más acción (Cyran et al., 2017) por ello interesa destacar y profundizar en la idea expuesta más arriba acerca de la experiencia y su influencia en actitudes más positivas hacia la discapacidad. No cabe duda de que la formación específica acerca de la discapacidad y el modelo inclusivo es una necesidad para lograr que dicha inclusión sea real ya que las actitudes pueden verse afectadas por la cantidad de preparación académica (Casebolt \& Hodge, 2010; Haegele, Hodge, Filho \& de Rezende, 2018; Jovanoviæ et al., 2014), estudiar materias afines a la discapacidad es un elemento positivo con el fin de mejorar las actitudes hacia la misma (Abellán, 2015). Pero más allá de la formación es necesaria la oportunidad de poner en práctica la teoría en contextos reales, los maestros en EF necesitan más oportunidades para trabajar (Hodge et al., 2009; Vickerman \& Coates, 2009), la calidad de la experiencia predice creencias positivas (Wang et al., 2015). Tindall, MacDonald, Carroll\& Moody (2015) demostraron el impacto de un programa de actividad física adaptado de 10 semanas sobre las actitudes y percepciones de 64 maestros irlandeses para enseñar a niños y jóvenes con discapacidades físicas, intelectuales y de aprendizaje. Los resultados revelaron un cambio positivo en la actitud y la percepción hacia la idea de inclusión y el trabajo con personas con discapacidad. En síntesis, la formación y las experiencias positivas previas son imprescindibles para que los docentes de EF exhiban una adecuada percepción de competencia, confianza y actitud positiva para la inclusión de alumnos con discapacidad (Reina et al., 2016).

\section{Conclusiones}

Es evidente que la actualidad y el futuro de la educación se liga a un modelo inclusivo basado en la calidad educativa y la igualdad de oportunidades para todo el alumnado independientemente de sus condiciones, particularidades o contexto. El área de EF cobra un papel relevante para facilitar la inclusión del alumnado con discapacidad, siendo esta área el escenario perfecto para la puesta en marcha de aprendizajes inclusivos y entornos cooperativos. Para este fin, ha de contarse con la buena disposición de los docentes de EF, teniendo en cuenta que su actitud se verá sin duda influida por su conocimiento previo de la discapacidad y necesidades del alumnado además de los recursos disponibles. Los resultados de este estudio muestran que los profesores participantes presentan actitudes moderadamente positivas hacia la inclusión de alumnos con discapacidad.

El estudio presentado no está exento de limitaciones, dado el carácter descriptivo y el reducido tamaño de la mues- 
tra no es posible realizar generalizaciones significativas. Asimismo, el instrumento de recolección de datos basado en datos auto-informados no está libre de sesgos. En vista de los resultados encontrados en esta investigación, y a pesar de las limitaciones descritas, es posible delinear al menos dos líneas de actuación futuras. La primera se refiere al propio estudio, será necesario en futuras investigaciones ampliar el tamaño de la muestra recopilando información de más docentes de EF contrastando la información con al menos dos instrumentos de recogida de datos. De este modo se podrán generalizar resultados además de determinar con exactitud las diferencias en función de las variables sociodemográficas. En este sentido, interesa indagar en las diferencias con respecto a la ubicación del centro y su relación con la disponibilidad de recursos. La segunda línea se refiere a la formación del profesorado de EF en lo que respecta a la atención de alumnos con discapacidad y programas de cambios de actitudes. Como ya se ha señalado con anterioridad el contacto con personas con discapacidad, la formación y el entrenamiento influyen en la mejora de las actitudes y es por tanto necesario diseñar formaciones encaminadas al fomento de la inclusión educativa.

\section{Referencias}

Abdi, E. (2017). Attitudes of students with learning disabilities toward participation in physical education: A teachers' perspective - qualitative examination. Proquest Llc,

Abellán, J. (2015). Actitudes hacia la discapacidad de los futuros maestros de Educación Física. Sportis, 1(3), 207-219. doi.org/ 10.17979/sportis.2015.1.3.1414

Allport, G. W. (1935), «Attitudes». En Carl Allanmore Murchison (Ed.): A handbookof social psychology. Winchester, MA: Clark University Press, pp.798-844.

Block, M. E., Kwon, E. H. \& Healy, S. (2016). Preparing future physical educators for inclusion: Changing the physical education teacher training program. Journal of the Brazilian Society for Adapted Motor Activity, 17(1), 9-12.

Cagran, B. \& Schmidt, M. (2011). Attitudes on Slovene teachers towards the inclusion of pupils with different types of special needs in primary school. Educational Studies, 37 (2), 171-195. doi.org/10.1080/03055698.2010.506319

Casebolt, K. M. \& Hodge, S. R. (2010). High school physical education teachers' beliefs about teaching students with mild to severe disabilities. Physical Educator, 67(3), 140.

Cyran, M., Kudlacek, M., Block, M., Malinowska-Lipien, I. \& Zyznawska, J. (2017). Attitudes of teachers towards inclusion of students with disabilities in physical education: Validity of the ATIPDPE-R instrument in polish cultural context. Acta Gymnica, 47(4), 171-179. doi.org/10.5507/ag.2017.020

Dias, P. C. \& Cadime, I. (2016). Effects of personal and professional factors on teachers' attitudes towards inclusion in preschool. European Journal of Special Needs Education, 31(1), 111-123. doi.org/10.1080/ 08856257.2015.1108040

Di Nardo, M., Kudláèek, M., Tafuri, D. \& Sklenaøíková, J. (2014). Attitudes of preservice physical educators toward individuals with disabilities at University Parthenope of Napoli. Acta Gymnica, 44(4), 211-221. doi.org/10.5507/ag.2014.022

Domínguez-Alonso, J., López-Castedo, A., Pino, M. R., \& Vázquez, E. (2015). Integración o inclusión: El dilema educativo en la atención a la diversidad. Revista Portuguesa de
Educação, 28(2), 31-50. doi.org/10.21814/rpe.7749

Felipe-Rello,C., Garoz, I. \& Tejero-Gonzalez, C.M (2018). Análisis comparativo del efecto de tres programas de sensibilización hacia la discapacidad en Educación Física (Comparative analysis of the effect of three Physical Education programs on awareness toward disability). Retos: Nuevas tendencias en Educación Física, Deportes y Recreación, 0(34), 258-262. Recuperado de https://recyt.fecyt.es/index.php/retos/article/ view/59889

Felipe-Rello, C., Garóz, I. \& Tejero-González, C.M. (2020). Cambiando las actitudes hacia la discapacidad: diseño de un programa de sensibilización en Educación Física (Changing attitudes towards disability: design of an awareness program in Physical Education). Retos: Nuevas tendencias en Educación Física, Deportes y Recreación, 37(37), 713-721. Recuperado de https:// recyt.fecyt.es/index.php/retos/article/view/69909

Fernández, J.M., Jiménez, F., Navarro, V. \& Sánchez, C.R. (2019). Cambios en el autoconcepto del alumnado con y sin discapacidad motriz a partir de una intervención docente inclusiva en Educación Física (Changes in self-concept of students with and without motor disabilities after an inclusive teaching intervention in P.E). Retos: Nuevas tendencias en Educación Física, Deportes y Recreación, 36(36), 138-145. Recuperado de https://recyt.fecyt.es/index.php/retos/article/view/ 67717

Fernández-Rivas, M. \& Espada, M. (2017). Evaluación de la actitud del profesorado de educación física ante alumnos con Necesidades Educativas Especiales. Sportis. Scientific Journal of School Sport, Physical Education and Psychomotricity, 3(3), 542-553. doi.org/10.17979/sportis.2017.3.3.2051

Garzón, P., Calvo, M. I. \& Orgaz, M.B. (2016). Inclusión educativa. Actitudes y estrategias del profesorado. Revista Española de Discapacidad (REDIS), vol. 4, núm. 2, pp. 25-45, en: https:/ /www.cedd.net/redis/index.php/redis/article/view/198/pdf_42

Gentil, M., Zurita, F., Gómez, V., Padial, R. \& Lara, A.J. (2019). Influencia de la práctica de actividad física en el autoconcepto de adolescentes (Influence of the practice of physical activity on the self-concept of adolescents). Retos: Nuevas tendencias en Educación Física, Deportes y Recreación, 36, 342-347. Recuperado de https://recyt.fecyt.es/index.php/retos/article/ viewFile/68852/43608

González, J. \& Cortés, M. R. (2016). Actitudes y creencias hacia la discapacidad en clases de educación física. Una cuestión educativa. Psychology, Society \& Education, 8(2), 105-120. doi.org/10.25115/psye.v8i2.454

Guillamón, A. R., García, E. \& Carrillo, P. J. (2019). Actividad física, condición física y autoconcepto en escolares de 8 a 12 años. Retos: Nuevas tendencias en Educación Física, Deportes y Recreación, 35, 236-241. Recuperado de: https:// recyt.fecyt.es/index.php/retos/article/view/64083/40966

González, I. \& Macías, D. (2018). La formación permanente como herramienta para mejorar la intervención del maestro de educación física con alumnado con discapacidad (Lifelong learning as a tool to improve physical education teachers' intervention with students with disabilities). Retos: Nuevas tendencias en Educación Física, Deportes y Recreación,33, 118-122. Recuperado de https://recyt.fecyt.es/index.php/retos/article/view/ 55056

Haegele, J. A., Hodge, S., Filho, P. J. \& de Rezende, L. G. (2018). Brazilian physical education teachers' attitudes toward inclusion before and after participation in a professional development workshop. European Physical Education Review, 24(1), 21. doi.org/10.1177/1356336X16662898

Hodge, S., Ammah, J. O., Casebolt, K. M., LaMaster, K., Hersman, 
B., Samalot Rivera, A. \& Sato, T. (2009). A diversity of voices: Physical education teachers' beliefs about inclusion and teaching students with disabilities. International Journal of Disability, Development and Education, 56(4), 401-419. doi.org/10.1080/ 10349120903306756

Hodge, S., Lieberman, L. \& Murata, N. (2017). Essentials of teaching adapted physical education: Diversity, culture, and inclusion. New York, Routledge. doi.org/10.4324/9781351217385

Hutzler, Y., Meier, S., Reuker, S. \& Zitomer, M. (2019). Attitudes and self-efficacy of physical education teachers toward inclusion of children with disabilities: A narrative review of international literature. Physical Education and Sport Pedagogy, 24(3), 249. doi.org/10.1080/ 17408989.2019.1571183

Jerlinder, K., Danermark, B. \& Gill, P. (2010). Swedish primaryschool teachers' attitudes to inclusion - the case of PE and pupils with physical disabilities. European Journal of Special Needs Education, 25(1), 45. doi.org10.1080/ 08856250903450830

Jovanoviæ, L., Kudláèek, M., Block, M. E. \& Djordjeviæ, I. (2014). Self-efficacy of pre-service physical education teacher toward teaching students with disabilities in general physical education classes in Serbia. European Journal of Adapted Physical Activity, 7(2).32-46. doi.org/10.5507/euj.2014.009

Morley, D., Bailey, R., Tan, J. \& Cooke, B. (2005). Inclusive Physical Education: teachers' views of including pupils with Special Educational Needs and/or disabilities in Physical Education. European Physical Education Review, 11 (1), 84107. doi.org10.1177/1356336x05049826

Mula,A. (dir.). (2002). Incidencia de las actitudes yexpectativas de alumnos y profesores sobre el desarrollo del programa de integración. Alicante: Universidad deAlicante.

Muñoz, A., Smith, E. \& Matos, M. (2020). Una experiencia de inclusión educativa en el aula de Expresión Corporal con alumnado Universitario (An Educational Inclusion Experience in a Body Expression course with undergraduate students). Retos: Nuevas tendencias en Educación Física, Deportes y Recreación, 37, 702-705. Recuperado de https://recyt.fecyt.es/ index.php/retos/article/view/68438

Novo-Corti, I., Muñoz-Cantero, J. M. \& Calvo-Babío, N. (2015). Los futuros docentes y su actitud hacia la inclusión de personas con discapacidad. Una perspectiva de género. Anales de Psicología/Annals of Psychology, 31(1), 155-171. doi.org/ 10.6018/analesps.31.1.163631

Pegalajar, M. C. \& Colmenero, M.J. (2014). Actitudes del docente de centros de educación especial hacia la inclusión educativa. Enseñanza \& teaching, 32 (2) 195-203. Recuperado de http:/

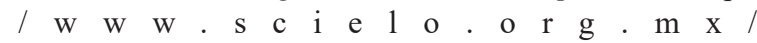
scielo.php?script $=$ sci_abstract $\&$ pid $=$ S 1607 40412017000100084\&lng=es\&nrm=iso

Rakap, S., Parlak-Rakap, A. \& Aydin, B. (2016). Investigation and comparison of Turkish and American preschool teacher candidates' attitudes towards inclusion of young children with disabilities. International Journal of Inclusive Education, 20(11), 1223-1237. doi.org/10.1080/ 13603116.2016 .1159254

Reich, L. M. (2012). Adapted physical education teachers' attitudes towards their students with hidden and visible disabilities. Unpublished Ed.D.Dissertation, California State University, California, United States.

Reina, R. y Ferriz, R. \& Roldán, A. (2019). Validation of a Physical Education Teachers' Self-Efficacy Instrument Toward Inclusion of Students With Disabilities. Frontiers in Psychology. 10, 110. 10.3389/fpsyg.2019.02169.
Reina, R., Santana, A., Montesdeoca, R. \& Roldán, A. (2019). Improving Self-Efficacy Towards Inclusion in In-Service Physical Education Teachers: A Comparison between Insular and Peninsular Regions in Spain. Sustainability. 11(20), 5824. 10.3390/su11205824

Rekaa, H., Hanisch, H. \& Ytterhus, B. (2019). Inclusion in physical education: Teacher attitudes and student experiences. A systematic review. International Journal of Disability, Development and Education, 66(1), 36. doi.org/10.1080/ 1034912X.2018.1435852

Ríos, M. (2009). La inclusión en el área de Educación Física en España. Análisis de las barreras para la participación y aprendizaje. Ágora para la Educación Física y el Deporte, 9, 83-114. Recuperado de http:/hdl.handle.net/11181/3837

Saloviita, T. \& Schaffus, T. (2016). Teacher attitudes towards inclusive education in Finland and Brandenburg, Germany and the issue of extra work. European Journal of Special Needs Education, 31(4), 458-471. doi.org/10.1080/ 08856257.2016.1194569

Sato, T. \& Hodge, S. (2009) Japanese physical educators' beliefs on teaching students with disabilities at urban high schools. Asia Pacific Journal of Education, 29(2), 159-177, doi: 10.1080/ 02188790902857164

Solís, P., Pedrosa, I. \& Fernández-Mateos, L.M. (2019). Assessment and interpretation of teachers' attitudes towards students with disabilities / Evaluación e interpretación de la actitud del profesorado hacia alumnos con discapacidad. Cultura y Educación, 31(3), 576-608, doi.org/ 10.1080/11356405.2019.1630955

Tindall, D., MacDonald, W., Carroll, E. \& Moody, B. (2015). Preservice teachers' attitudes towards children with disabilities: An irish perspective. European Physical Education Review, 21(2), 206. doi.org/10.1177/1356336X14556861

Torres, J. A. \& Fernández, J. M. (2015). Promoviendo escuelas inclusivas: Análisis de las percepciones y necesidades del profesorado desde una perspectiva organizativa, curricular y de desarrollo profesional. Revista Electrónica Interuniversitaria de Formación del Profesorado, 18(1), 177-200. doi.org/ 10.6018/reifop.18.1.214391

Triandis, H. C. (1971). Attitude and attitude change. New York, NY: John Wiley and Sons.

UNESCO (2005). Guidelines for inclusión: ensuring access to education for all. París: Unesco

Urton, K., Wilbert, J. \& Hennemann, T. (2014). Attitudes towards inclusion and self-efficacy of principals and teachers. Learning Disabilities - A Contemporary Journal, 12(2), 151-168.

Valencia-Peris, A., Mínguez-Alfaro, P. \& Martos-García, D. (2020) La formación inicial del profesorado de Educación Física: una mirada desde la atención a la diversidad (Pre-service Physical Education Teacher Education: a view from attention to diversity). Retos: Nuevas tendencias en Educación Física, Deporte y Recreación, 37, 597-604. Recuperado de https:// recyt.fecyt.es/index.php/retos/article/download/74180/45765

Vickerman, P. \& Coates, J. K. (2009). Trainee and recently qualified physical education teachers' perspectives on including children with special educational needs. Physical Education and Sport Pedagogy, 14(2), 137-153. doi.org/10.1080/ 17408980802400502

Wang, L., Qi, J. \& Wang, L. (2015). Beliefs of Chinese physical educators on teaching students with disabilities in general physical education classes. Adapted Physical Activity Quarterly, 32(2), 137-155. doi.org/10.1123/apaq.2014-0140 